Breast feeding

\title{
Communicating the benefits of breast feeding
}

\section{Ken K Ong, Nita Forouhi}

\section{Perspective on the paper by Akobeng and Heller (see page 483)}

$t$ is hopefully clear to readers of the Archives that exclusive breast feeding has several important health benefits and should be encouraged. But what is the best way to convey the extent of its benefits to an individual mother, or to a health care organisation deciding on the allocation of resources for health promotion?

\section{MEASURES OF ASSOCIATION AND BENEFIT}

Clinicians and research scientists are usually more familiar with, and tend to favour, measures of association such as relative risks (or odds ratios) and measures of impact such as the population attributable risk (PAR), which is the proportion of a disease that, assuming causality, could be avoided if that risk factor were removed. However, the size of a benefit, whether to the individual or to the population, is better measured in terms of actual risks or risk differences, because those measures also take into account the actual likelihood of the disease outcome. For example, exclusive breast feeding may cause a greater reduction in cases of coeliac disease (44\%) than of asthma (23\%), but because asthma is much more common, the numbers of children who would avoid asthma is far greater than for coeliac disease.

In this issue of the Archives, Akobeng and Heller have utilised a new calculation of population impact, which was recently described by their group in Manchester, ${ }^{1}$ to demonstrate the effect of breast feeding on the numbers of cases with asthma, obesity and coeliac disease that could potentially be avoided. ${ }^{2}$ While the phrase "population impact number of eliminating a risk factor over a time period" (PINER- $t$ ) is unlikely to roll off the tongues of community midwives, health visitors or paediatricians, the result is easy to interpret as "the number of cases of a disease that might be avoided by eliminating the risk factor". It is also conceptually straightforward (in essence it is simply the PAR multiplied by the total number of cases in a population). Figure 1 graphically illustrates the PIN-ER-t results from
Akobeng and Heller's paper, and shows its relationship with the PAR.

\section{ASSUMPTIONS}

Are the PIN-ER- $t$ results of Akobeng and Heller $^{2}$ true? Could we really avoid 33100 cases of asthma, 13639 cases of obesity and 2655 cases of coeliac disease up to age 7-9 years among children born in 2002 in England and Wales through breast feeding?

The calculations are certainly valid. However, they depend on various assumptions. The first is that it is possible to increase the prevalence of breast feeding up to age 3 months from the current $30 \%$ to $100 \%$. Fortunately, the authors also provide numbers for a range of more realistic interim targets. Secondly, the numbers are dependent on historical rates of disease, although the numerical impact will likely increase as asthma and obesity are becoming more prevalent. However, the main assumption is that the association between breast feeding and disease benefits seen in observational studies is entirely causal. This is unlikely, as in the UK, mothers who breast feed differ from other mothers with regard to social class, education and

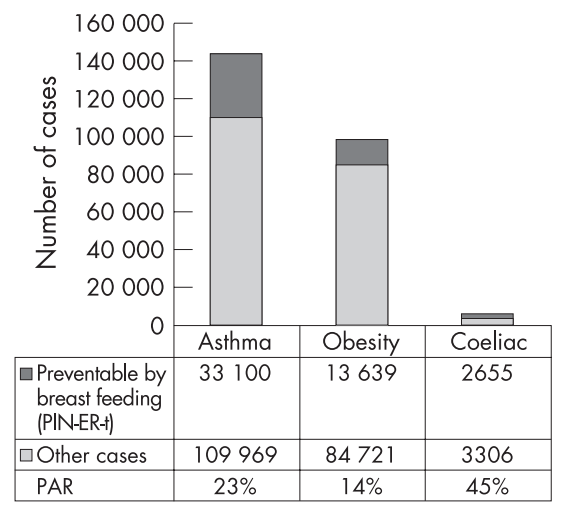

Figure 1 Numbers of cases of asthma, obesity and coeliac disease diagnosed up to age 7-9 years among the 596122 children born in 2002 in England and Wales, including the population attributable risk (PAR) and actual numbers of cases attributable to formula milk feeding. Data are taken from Akobeng and Heller. ${ }^{2}$ various other diet and lifestyle choices. Any differences in the incidence of disease in their children could therefore be partly due to (or confounded by) any of those other differences. For example, apparent benefits of breast feeding on childhood intelligence are abolished if one accounts for baseline differences in mothers' education and other confounders. ${ }^{4}$

The only randomised trial of breast feeding promotion, involving 17000 infants in Belarus, increased rates of exclusive breast feeding at age 3 months from $6 \%$ to $43 \%$, and led to significant reductions in the risks of gastrointestinal tract infections and atopic eczema. ${ }^{5}$ Even in the absence of randomised trial data for the other disease outcomes, most readers would support the World Health Organization and Department of Health's recommendations that exclusive breast feeding for the first 6 months of life is the best form of infant nutrition. ${ }^{6}$ However, reliance on the observational data for asthma, obesity and coeliac disease will likely overestimate the true benefits of increasing breast feeding rates.

\section{INCREASING THE PREVALENCE OF BREAST FEEDING IN THE UK}

Notwithstanding the many assumptions in Akobeng and Heller's calculations of the population impact of low breast feeding rates, their findings are important and should not be ignored. In light of these and other widespread benefits of breast feeding such as on type 1 diabetes and maternal obesity, why do rates of breast feeding in the UK remain among the lowest in Western Europe and indeed worldwide?

The NHS Plan 2003-06 aimed to increase breast feeding initiation rates by $2 \%$ per year. As important a goal would be to increase the duration of breast feeding in those mothers who start. The UNICEF UK Baby Friendly Initiative has helped to increase the initiation of breast feeding in the UK to around $70 \%$. However, less than $50 \%$ breast feed up to 1 month, and only around $30 \%$ continue to 3 months, which may be around the duration required to achieve the above benefits. ${ }^{2}$ The apparent lack of effect of the Baby Friendly Initiative to increase breast feeding duration even up to 1 month in the Millennium Cohort Study ${ }^{7}$ should not be surprising as in the UK there are 51 maternity hospitals with full Baby Friendly accreditation compared to only seven community health care facilities. ${ }^{8}$

Four further voluntary organisations provide supplementary support to breast feeding women in the UK. These are the Association of Breastfeeding Mothers, the Breastfeeding Network, La Leche League 
and the National Childbirth Trust, each with its own particular philosophy and aims. Research is needed to determine the availability and uptake of such support by women, and their impact on prolonging breast feeding. Future research should also try to clarify the duration of breast feeding required to achieve each benefit, whether breast feeding needs to be exclusive, and whether the apparent benefits might be due to other related feeding behaviours, such as the timing, or type, of weaning foods.

\section{CONCLUSIONS}

Healthcare providers should benefit from PIN-ER- $t$ and the summary epidemiological data reported by Akobeng and Heller $^{2}$ to calculate the numbers of disease cases that might be avoided. Strategic Health Authorities and Primary Care Trusts could use this information to estimate the cost effectiveness of community-based interventions to increase the initiation and duration of breast feeding within their individual populations.

What about the individual mother deciding whether or not to breast feed? Effective health communication relies on individual understanding and the availability of different methods of communication. Some mothers may be swayed by odds ratios, percentages or other risk estimates. However, health promotion often needs to target groups with lower levels of health literacy, and to translate such figures into everyday realities. Individual case reports are increasingly used by the news media and charities to convey the otherwise "inconceivable" impact of events that affect hundreds or thousands of people. ${ }^{9}$ Describing the benefits of breast feeding for the infant and mother, together with the numbers of cases of disease conditions that might be avoided by breast feeding (using concepts such as the PINER- $t$ ) could be a potential novel effective health communication strategy. It might just get the message through.

Arch Dis Child 2007;92:471-472.

doi: 10.1136/adc.2006.113738

\section{Authors' affiliations}

Ken K Ong, Nita Forouhi, MRC Epidemiology Unit, Cambridge, UK

Correspondence to: Dr Ken Ong, MRC Epidemiology Unit, Strangeways Research Laboratory, Worts Causeway, Cambridge CB1 8RN, UK; ken.ong@mrc-epid.cam.ac.uk
Competing interests: None.

\section{REFERENCES}

1 Heller RF, Buchan I, Edwards R, et al. Communicating risks at the population level: application of population impact numbers. BMJ 2003;327: 1162-5.

2 Akobeng AK, Heller RF. Assessing the population impact of low rates of breast feeding on asthma, coeliac disease and obesity: the use of a new statistical method. Arch Dis Child 2007;92:483-5.

3 Emmett P, North K, Noble S. Types of drinks consumed by infants at 4 and 8 months of age: a descriptive study. The ALSPAC Study Team. Public Health Nutr 2000;3:211-7.

4 Der G, Batty GD, Deary IJ. Effect of breast feeding on intelligence in children: prospective study, sibling pairs analysis, and meta-analysis. BMJ 2006;333:945

5 Kramer MS, Chalmers B, Hodnett ED, et al. Promotion of Breastfeeding Intervention Trial (PROBIT): a randomized trial in the Republic of Belarus. JAMA 2001;285:413-20.

6 Department of Health. Infant feeding recommendation. http://www.dh.gov.uk/ assetRoot/04/09/69/99/04096999.pdf (accessed 21 March 2007).

7 Bartington S, Griffiths $U$, Tate AR, et al. Are breastfeeding rates higher among mothers delivering in Baby Friendly accredited maternity units in the UK? Int J Epidemiol 2006;35: 1178-86.

8 UNICEF. The Baby Friendly Initiative. http:// www.babyfriendly.org.uk/ (accessed 21 March 2007).

9 Oxfam. Tsunami crisis. What it means to have a home. http://www.oxfam.org.uk/what_we_do/ emergencies/country/asiaquake/oneyearon/ shelter/indonesia/index.htm (accessed 21 March 2007)

\section{Constipation and incontinence in childhood: two sides of the same coin?}

\section{Graham Clayden, Anne Wright}

\section{Perspective on the paper by Loening-Baucke (see page 486)}

$\mathrm{T}$ he relationship between constipation and faecal and urinary retention is very familiar to paediatricians, but the prevalence of these distressing symptoms appears to vary in the medical literature. We will explore the background to the development of continence and how this may be disturbed by constipation.

\section{REFLECTION ON THE ARTICLE}

As Loening-Baucke stresses in the article, the problem with literature review has been the lack of an agreed set of definitions for constipation. The Paris consensus has improved on the Rome II definitions and she has shown how valuable it is to apply these even in a retrospective notes survey. ${ }^{1}$ Her study also avoids the problem of extrapolating data from a referral centre to the whole community. As with any epidemiological study there may be characteristics of the community being studied that limit its generalisability. LoeningBaucke's population is based on a university and so may not have an average socio-economic profile. A population based study in the UK showed a prevalence in 7.5 year old children of infrequent daytime faecal incontinence (no more than once a week) of $6.8 \%$ and of severe incontinence (at least daily) of $0.8 \%{ }^{2}$

\section{IMPORTANCE OF CONTINENCE AND KNOWING ITS EPIDEMIOLOGY}

The acquisition of continence is an essential element for children to be able to fully integrate with others and to access most public activities. Delays or disruptions in achieving continence will severely limit social life and continence is an important marker of quality of life at any age beyond toddlerhood. Unless we have a reasonable understanding of the prevalence of incontinence, it is impossible to focus resources on this important problem. However, a clear measure is extremely difficult. A degree of incontinence for one child or family may be so acceptable that it is never mentioned but for another may be the reason for referral for medical help. Social constraints may also be driving factors for help being sought. For example, in the UK there has been an increase in the availability of preschool experience for children. If a preschool placement is dependent on a child being pot trained and out of nappies, a parent may seek medical help for a 2.5 year old who cannot reliably use the pot who would otherwise have been reassured to know that probably a half of healthy children of that age are equally unreliable about keeping their pants clean and dry.

\section{NORMAL DEVELOPMENT OF CONTINENCE}

To achieve continence the child must be able to perceive sensation arising from 